\title{
Positive Newtonian Capacity and Stationary Viscous Incompressible Flow
}

\section{VICTOR L. SHAPIRO}

\author{
Communicated by the Editors
}

1. Introduction. Operating in Euclidean 3-space, we shall say $\mathbf{v}=\left(v_{1}, v_{2}, v_{3}\right)$ is a stationary viscous incompressible flow in an open set $\Omega$ if there is a pressure $p$ such that

(1.1) $\quad \mathrm{v}$ is in $C^{2}(\Omega)$, and $p$ is in $C^{1}(\Omega)$;

(1.2) $\quad v_{j}, \partial v_{j} / \partial x_{k}$, and $p$ are in $L^{2}(\Omega)$ for $j, k=1,2,3$;

(1.3) there is a non-zero constant $\nu$ such that in $\Omega$ the following equations are satisfied:

$$
\left\{\begin{array}{r}
\nu \Delta \mathrm{v}-(\mathrm{v} \cdot \nabla) \mathrm{v}-\nabla p=0 \\
\operatorname{div} \mathbf{v}=\mathbf{0} .
\end{array}\right.
$$

Let $Z$ be a closed set contained in the interior of the unit ball, i.e., $Z \subset B(0,1)$ where $B(x, r)$ designates the open 3-ball with center $x$ and radius $r$. We shall say $Z$ is a removable set for stationary viscous incompressible flows if the following holds:

If $\mathbf{v}$ and $p$ meet (1.1), (1.2), and (1.3) with $\Omega=B(0,1)-Z$, then $\mathbf{v}$ and $p$ can be defined at the points of $Z$ so that $\mathrm{v}$ and $p$ meet (1.1), (1.2), and (1.3) with $\Omega=$ $B(0,1)$.

In this paper, we intend to establish the necessary part of the following theorem:

Theorem. A necessary and sufficient condition that a closed set $Z \subset B(0,1)$ be removable for stationary viscous incompressible flows is that $Z$ be of Newtonian capacity zero.

The sufficiency part of the above theorem is an immediate corollary of [5], [6], and [2, p. 10]. 
The notion of Newtonian capacity zero for closed sets is a classical concept, but we give it here for completeness. The closed set $Z \subset B(0,1)$ is of Newtonian capacity zero if it is not of positive Newtonian capacity. $Z$ is a set of positive Newtonian capacity if there exists a non-negative Borel measure $\mu$ such that $\mu[B(0,1)-Z]=0, \mu(Z)=1$, and

$\int_{Z}|x-y|^{-1} d \mu(y)$ is a uniformly bounded function of $x$ in $B(0,1)-Z$.

The main result of this paper is obtained via a type of existence theorem which does not appear to be very prevalent in the general area of nonlinear partial differential equations. To obtain this theorem, we combine the functional analytic methods previously used in dealing with the Navier-Stokes equations (see [2, Chapter 5]) with various techniques used in the area of trigonometric series and its applications (see [3], [4], [5], and [6]).

2. Flows and multiple Fourier Series. To establish the necessary part of the above theorem, we shall need some lemmas connecting multiple Fourier series with the nonlinear stationary Navier-Stokes equations defined in (1.3).

We shall use the following notation:

$T_{3 .}=\left\{x:-\pi \leqq x_{i}<\pi, j=1,2,3\right\} ; m$ will designate an integral lattice point; for a function $g$ in $L^{1}\left(T_{3}\right)$, we shall set

$$
g^{\wedge}(m)=(2 \pi)^{-3} \int_{T_{3}} g(x) e^{-i(m, x)} d x .
$$

Also, $(x, y)$ will designate the usual inner product, and $(x, x)^{1 / 2}$ will be designated by $|x|$.

In order to establish our result, we shall have to introduce a number of functions.

We first introduce the function $q_{i}(x)$ defined in $E_{3}$ as follows:

$$
q_{i}(x)=\lim _{t \rightarrow 0} \sum_{m \neq 0} i m_{j} e^{i(m, x)-|m| t}|m|^{-2} .
$$

From [3, p. 72], we obtain that the following properties prevail for $j=1,2,3$ :

$$
\begin{aligned}
& q_{i}(x) \text { is in } L^{1}\left(T_{3}\right) ; \\
& q_{i}{ }^{\wedge}(m)=i m_{j} /|m|^{2} \text { for } m \neq 0 ; \\
& q_{i}^{\wedge}(0)=0 .
\end{aligned}
$$

In a similar manner, we introduce the function $H(x)$ defined in $E_{3}$ as follows:

$$
H(x)=\lim _{i \rightarrow 0} \sum_{m \neq 0} e^{i(m, x)-|m| t}|m|^{-2} .
$$

From [3, p. 72], we obtain that the following properties prevail:

$$
\begin{aligned}
& H(x) \text { is in } L^{1}\left(T_{N}\right), \\
& H^{\wedge}(m)=|m|^{-2} \text { for } m \neq 0 \text { and } \\
& H^{\wedge}(0)=0 .
\end{aligned}
$$


From [3, p. 72], we obtain also that

$$
H(x) \text { and } q_{j}(x) \text { are in } C^{\infty}\left[E_{3}-\bigcup_{m}\{2 \pi m\}\right],
$$

and that

$$
\triangle H(x)=1 \text { and } \triangle q_{i}(x)=0 \text { in } E_{3}-\bigcup_{m}\{2 \pi m\} .
$$

Furthermore, we obtain from this same reference that there are positive constants $\alpha, \alpha_{i}, \beta$, and $\beta_{i}$ such that

$$
\begin{aligned}
\left.|H(x)-\alpha| x\right|^{-1} \mid & \leqq \beta \\
\left.\left|q_{j}(x)+\alpha_{j} x_{i}\right| x\right|^{-3} \mid & \leqq \beta_{i} \quad \text { for } \quad x \text { in } T_{3}-\{0\} .
\end{aligned}
$$

Next, we set

$$
G(x)=-\sum_{m \neq 0} e^{i(m, x)}|m|^{-4}
$$

and observe that the series in (2.9) converges absolutely. Consequently, $G(x)$ is a continuous periodic function. We conclude from [3, Lemma 7], (2.4), (2.6), and well-known facts concerning subharmonic functions that

$$
G(x) \text { is in } C^{\infty}\left[E_{3}-\bigcup_{m}\{2 \pi m\}\right]
$$

and furthermore that

$$
\triangle G(x)=H(x) \text { in } E_{3}-\bigcup_{m}\{2 \pi m\} .
$$

From (2.8) and (2.11), we conclude with $\alpha$ the constant defined in (2.8) that

$$
G(x)-2^{-1} \alpha|x| \text { is in } C^{\infty}[B(0,2)] .
$$

Next, motivated by $[2$, p. 51], we set for $j, k=1,2,3$,

$$
\nu u_{k}^{j}(x)=\lim _{t \rightarrow 0} \sum_{m \neq 0}\left[-\delta_{k}^{i}+m_{i} m_{k}|m|^{-2}\right]|m|^{-2} e^{i(m, x)-|m| t},
$$

where $\delta_{k}{ }^{i}$ designates the Kronecker $\delta$ and, observe from (2.10) and [4, Lemma 2] that the above limit exists and is finite in $E_{3}-\bigcup_{m}\{2 \pi m\}$. (It clearly exists and is equal to $-\infty$ for $x=2 \pi m$ and $j=k$.)

From (2.9), (2.10), (2.11), and [4, Lemma 2], we see that

$$
\nu u_{k}^{i}(x)=-\delta_{k}^{i} H(x)+\partial^{2} G(x) / \partial x_{i} \partial x_{k} \text { for } x \text { in } E_{3}-\bigcup_{m}\{2 \pi m\},
$$

and consequently from (2.10) and (2.12), we obtain that

$$
\begin{aligned}
& \nu u_{k}^{i} \text { is in } L^{1}\left(T_{3}\right), \text { and } \\
& \nu u_{k}^{i^{\wedge}}(m)=\left[-\delta_{k}{ }^{i}+m_{i} m_{k}|m|^{-2}\right]|m|^{-2} \text { for } m \neq 0 \\
& \nu u_{k}^{i^{\wedge}}(0)=0 .
\end{aligned}
$$

Next, we say that $g$ is in $W_{2}{ }^{1}\left(T_{3}\right)$ if $g$ is in $L^{2}\left(T_{3}\right)$ and if $\sum\left|g^{\wedge}(m)\right|^{2}|m|^{2}<\infty$. 
We shall refer to the function in $L^{2}\left(T_{3}\right)$ whose Fourier series is $\sum i m_{j} g^{\wedge}(m) e^{i(m, x)}$ by $g_{x_{i}}$. It is clear that $\int_{T_{3}} g(x) \psi_{x_{i}}(x) d x=-\int_{T_{3}} g_{x_{i}}(x) \psi(x) d x$ if $\psi$ is a periodic function (of period $2 \pi$ in each variable) which is in $C^{\infty}\left(E_{3}\right)$.

The following lemma then prevails:

Lemma 1. Let $g$ be in $W_{2}{ }^{1}\left(T_{3}\right)$ and suppose $g^{\wedge}(0)=0$. Then there is an absolute constant $K$, independent of $g$, such that

$$
\int_{T_{3}} g^{4} d x \leqq K\left\{\int_{T_{3}} g^{2} d x\right\}^{1 / 2}\left\{\int_{T_{3}}|\nabla g|^{2} d x\right\}^{3 / 2} .
$$

Proof. To establish the lemma, we define for $t>0$

$$
g(x, t)=\sum_{m} g^{\wedge}(m) e^{i(m, x)-|m| t},
$$

and observe that $g(x, t)$ is a periodic function in $C^{\infty}\left(E_{3}\right)$.

Next, we extend $g$ by periodicity of period $2 \pi$ in each variable to all of $E_{3}$ and observe from [3, pp. 55-6] that

$$
\lim _{t \rightarrow 0} g(x, t)=g(x) \quad \text { almost everywhere in } E_{3} .
$$

Also we have that for $t>0$,

$$
\begin{gathered}
\int_{T_{3}}|g(x, t)|^{2} d x \leqq \int_{T_{3}}|g(x)|^{2} d x \\
\int_{T_{3}}|\nabla g(x, t)|^{2} d x \leqq \int_{T_{3}}|\nabla g(x)|^{2} d x .
\end{gathered}
$$

Furthermore since $g^{\wedge}(0)=0$,

$$
\begin{gathered}
\int_{T_{3}}|g(x, t)|^{2} d x \leqq \int_{T_{3}}|\nabla g(x, t)|^{2} d x \\
\int_{T_{3}}|g(x)|^{2} d x \leqq \int_{T_{3}}|\nabla g(x)|^{2} d x .
\end{gathered}
$$

Next, we choose a function $\lambda(x)$ with the following properties:

$$
\begin{aligned}
& \lambda=1 \text { in } B(0,1) \\
& \lambda=0 \text { in } E_{3}-B(0,2) \\
& \lambda \text { is in } C^{\infty}\left(E_{3}\right) \text { and } 0 \leqq \lambda(x) \leqq 1 \text { for all } x .
\end{aligned}
$$

For $x^{0}$, a given point in $E_{3}$, we set $\psi(x)=\lambda\left(x-x^{0}\right)$. Then from [2; Lemma 2 , p. 9], we have

$$
\begin{aligned}
\int_{B\left(x^{0}, 1\right)} & |g(x, t)|^{4} d x \leqq \int_{B\left(x^{0}, 2\right)}|\psi(x) g(x, t)|^{4} d x \\
& \leqq 4\left\{\int_{B\left(x^{0}, 2\right)}|\psi(x) g(x, t)|^{2} d x\right\}^{1 / 2}\left\{\int_{B\left(x^{0}, 2\right)}|\nabla \psi(x) g(x, t)|^{2} d x\right\}^{3 / 2}
\end{aligned}
$$


Observing that $|\nabla \lambda(x)|$ is uniformly bounded in $E_{3}$, we conclude from (2.17), (2.18), (2.19), and (2.20) that there is a constant $K^{\prime}$, independent of $x^{0}$ and $g$, such that

$$
\int_{B\left(x^{0}, 1\right)}|g(x, t)|^{4} d x \leqq K^{\prime}\left\{\int_{T_{3}} g^{2}(x) d x\right\}^{1 / 2}\left\{\int_{T_{3}}\left(\left.\nabla g(x)\right|^{2} d x\right\}^{3 / 2} .\right.
$$

The conclusion to the lemma then follows immediately from Fatou's lemma, (2.16), (2.21), and the fact that a finite number of unit three-balls cover $T_{3}$.

Next, we observe that the following lemma holds:

Lemma 2. Let $g$ be in $W_{2}{ }^{1}\left(T_{3}\right)$ and suppose $g^{\wedge}(0)=0$. Then there is an absolute constant $K$, independent of $g$, such that

$$
\int_{T_{3}} g^{6} d x \leqq K\left\{\int_{T_{3}}|\nabla g|^{2} d x\right\}^{3} .
$$

The proof of Lemma 2 follows from [2, Lemma 3, p. 10] in a manner similar to the proof of Lemma 1 . We leave the details to the reader.

Next, we let ${ }_{0} W_{2}{ }^{1}\left(T_{3}\right)$ designate the Hilbert space introduced in Lemmas 1 and 2, i.e.,

$$
{ }_{0} W_{2}{ }^{1}\left(T_{3}\right)=\left\{g: g \text { in } W_{2}{ }^{1}\left(T_{3}\right) \text { and } g^{\wedge}(0)=0\right\}
$$

where the inner product $\langle g, h\rangle$ is defined by

$$
\langle g, h\rangle=(2 \pi)^{-3} \int_{T_{3}}\left(g_{x_{1}} h_{x_{1}}+g_{x_{2}} h_{x_{3}}+g_{x_{3}} h_{x_{3}}\right) d x \text { for } g \text { and } h \text { in }{ }_{0} W_{2}^{1}\left(T_{3}\right) \text {. }
$$

The following lemma (see [2, p. 13]) then prevails:

Lemma 3. Let $g$ and $g_{k}$ be in ${ }_{0} W_{2}{ }^{1}\left(T_{3}\right)$ for $k=1,2, \cdots$. Suppose $g_{k}$ is weakly convergent to $g$ in ${ }_{0} W_{2}{ }^{1}\left(T_{3}\right)$. Then

$$
\lim _{k \rightarrow \infty} \int_{T_{3}}\left|g-g_{k}\right|^{4} d x=0 .
$$

Proof. With no loss in generality, we can suppose that $g=0$. Then it follows from the Banach-Steinhaus theorem that there is a constant $M$ such that

$$
\sum_{m}\left|g_{k}{ }^{\wedge}(m)\right|^{2}|m|^{2} \leqq M \text { for } k=1,2, \cdots \text {. }
$$

Also we have from weak convergence to zero that

$$
\lim _{k \rightarrow \infty} \hat{g}_{k}{ }^{\wedge}(m)=0 \text { for every } m \text {. }
$$

Next, we observe that

$$
\sum_{m}\left|g_{k}{ }^{\wedge}(m)\right|^{2} \leqq \sum_{|m| \leqq N}\left|g_{k}{ }^{\wedge}(m)\right|^{2}+N^{-2} \sum_{N \leqq|m|}\left|g_{k}{ }^{\wedge}(m)\right|^{2}|m|^{2} .
$$


We consequently obtain from (2.26), (2.27), and (2.28) that

$$
\limsup _{k \rightarrow \infty} \sum_{m}\left|g_{k}{ }^{\wedge}(m)\right|^{2} \leqq M N^{-2} \text {. }
$$

Since $N$ is arbitrary, we conclude that

$$
\lim _{k \rightarrow \infty} \int_{T_{3}}\left|g_{k}\right|^{2} d x=0 .
$$

On the other hand from Lemma 1 and (2.26) we obtain that there is a constant $K$ such that

$$
\int_{T_{3}} g_{k}{ }^{4} d x \leqq K\left\{\int_{T_{3}} g_{k}{ }^{2} d x\right\}^{1 / 2} M^{3 / 2} \text { for } k=1,2, \cdots
$$

The conclusion to the lemma then follows immediately from this last fact and (2.29).

Next, we define $\mathfrak{D}\left(T_{3}\right)$ as follows:

$$
\begin{aligned}
D\left(T_{3}\right)= & \left\{\psi: \psi \text { is in } C^{\infty}\left(E_{3}\right), \text { and } \psi \text { is periodic of period } 2 \pi\right. \\
& \text { in each variable }\} .
\end{aligned}
$$

The following lemma then holds:

Lemma 4. Let $f_{j}$ be in ${ }_{0} W_{2}{ }^{1}\left(T_{3}\right) \cap \mathfrak{D}\left(T_{3}\right)$ for $j=1$, 2, 3. Set

$$
v_{j}(x)=(2 \pi)^{-3} \sum_{k=1}^{3} \int_{T_{3}} u_{j}{ }^{k}(x-y) f_{k}(y) d y \text { for } j=1,2,3
$$

and

$$
p(x)=(2 \pi)^{-3} \sum_{k=1}^{3} \int_{T_{3}} q_{k}(x-y) f_{k}(y) d y .
$$

Then $p(x)$ and $v_{j}(x)$ are in ${ }_{0} W_{2}{ }^{1}\left(T_{3}\right) \cap D\left(T_{3}\right)$ for $j=1,2,3$ and

$$
\nu \Delta v_{i}-\partial p / \partial x_{i}=f_{i}, \quad j=1,2,3, \quad \sum_{i=1}^{3} \partial v_{i} / \partial x_{j}=0 .
$$

Proof. From (2.3) and $\left(2.14^{\prime}\right)$, we see that the integrals on the right hand side of (2.31) are well-defined and that

$$
\int_{T_{3}} u_{i}^{k}(x-y) f_{k}(y) d y=\int_{T_{3}} u_{i}^{k}(y) f_{k}(x-y) d y
$$

and

$$
\int_{T_{3}} q_{k}(x-y) f_{k}(y) d y=\int_{T_{3}} q_{k}(y) f_{k}(x-y) d y
$$


As a consequence, $p(x)$ and $v_{j}(x)$ are in $D\left(T_{3}\right)$. Also

$$
\begin{aligned}
v_{j}{ }^{\wedge}(m) & =\sum_{k=1}^{3}{u_{j}{ }^{\wedge}{ }^{\wedge}(m) f_{k}{ }^{\wedge}(m),}_{j}(m=1,2,3, \\
p^{\wedge}(m) & =\sum_{k=1}^{3}{q_{k}}^{\wedge}(m) f_{k}{ }^{\wedge}(m) .
\end{aligned}
$$

From (2.34), we see that ${v_{i}}^{\wedge}(0)=p^{\wedge}(0)=0$; consequently, $v_{i}$ and $p$ are also in ${ }_{0} W_{2}^{1}\left(T_{3}\right)$.

Next, from (2.3), (2.13), and (2.34), we observe that for $m \neq 0$,

$$
-\nu|m|^{2} v_{i}^{\wedge}(m)-i m_{i} p^{\wedge}(m)=f_{i}^{\wedge}(m), \quad j=1,2,3,
$$

and

$$
\sum_{i=1}^{3} i m_{j} v_{i}^{\wedge}(m)=0
$$

(2.33) follows from these facts and the fact that all derivatives of $p$ and $v_{i}$ can be obtained by differentiating their Fourier series under the summation sign. The proof of the lemma is therefore complete.

Lemma 5. Let $f_{j}$ be in ${ }_{0} W_{2}{ }^{1}\left(T_{3}\right)$ for $j=1,2$, 3. Let $v_{i}$ and $p$ be defined respectively by (2.31) and (2.32). Then $v_{i}$ and $p$ are in ${ }_{0} W_{2}{ }^{1}\left(T_{3}\right)$ and

$$
\begin{gathered}
\int_{T_{3}}\left[\nu v_{j} \Delta \psi+p \psi_{x_{j}}\right] d x=\int_{T_{3}} f_{j} \psi d x \\
\int_{T^{13}}\left[v_{1} \psi_{x_{1}}+v_{2} \psi_{x_{2}}+v_{3} \psi_{x_{3}}\right] d x=0 \text { for all } \psi \text { in } D\left(T_{3}\right) .
\end{gathered}
$$

Proof. (2.34) holds as before, and it is immediate from this that $v_{i}$ and $p$ are in ${ }_{0} W_{2}^{1}\left(T_{3}\right)$.

To establish (2.35), we introduce $v_{i}(x, t), f_{i}(x, t)$, and $p(x, t)$ for $t>0$ in a manner analogous to (2.15). But then the analogue of (2.34) holds for these functions, and consequently the analogue of (2.33) is also true. We obtain therefore that (2.35) holds when $v_{i}(x), p(x)$, and $f_{i}(x)$ are replaced respectively by $v_{j}(x, t), p(x, t)$, and $f_{i}(x, t)$. But, as is well-known [3; Remark 2, p. 76],

$$
\begin{aligned}
& \int_{T_{3}}\left|v_{j}(x, t)-v(x)\right| d x \rightarrow 0 \quad \text { as } \quad t \rightarrow 0, \\
& \int_{T_{3}}|p(x, t)-p(x)| d x \rightarrow 0 \text { as } t \rightarrow 0, \\
& \int_{T_{3}}\left|f_{i}(x, t)-f_{i}(x)\right| d x \rightarrow 0 \text { as } t \rightarrow 0,
\end{aligned}
$$

and the conclusion of the lemma follows. 
Next, we introduce the Hilbert space $\mathcal{H}\left(T_{3}\right)$. We say $\mathbf{v}=\left(v_{1}, v_{2}, v_{3}\right)$ is in $\operatorname{Fe}\left(T_{3}\right)$ if $v_{i}$ is in ${ }_{0} W_{2}{ }^{1}\left(T_{3}\right)$ for $j=1,2,3$ and if furthermore

$$
v_{1 x_{1}}+v_{2 x_{2}}+v_{3 x_{3}}=0 \text {. }
$$

The inner product $[\mathbf{v}, \mathbf{w}]$ in $\mathfrak{H C}\left(T_{3}\right)$ is defined as follows:

$$
[\mathbf{v}, \mathbf{w}]=\sum_{j=1}^{3}\left\langle v_{i}, w_{i}\right\rangle
$$

where $\left\langle v_{i}, w_{i}\right\rangle$ is defined by (2.24).

Next, we observe from Lemma 2 that for $v$ in $\mathfrak{H}\left(T_{3}\right), v_{j}$ is in $L^{6}\left(T_{3}\right)$ for $j=$ $1,2,3$. Consequently as in $\left[2\right.$, p. 116], we obtain that for fixed $\mathbf{v}$ in $\mathfrak{H C}\left(T_{3}\right)$ the expression

$$
(2 \pi)^{-3} \sum_{j=1}^{3} \sum_{k=1}^{3} \int_{T_{3}} v_{k} v_{j} \phi_{j x_{k}} d x
$$

defined for all $\phi$ in $\mathfrak{F}\left(T_{3}\right)$ gives rise to a bounded linear functional on $\mathfrak{H C}\left(T_{3}\right)$.

Consequently, there exists an element $A \mathbf{v}$ in $\mathfrak{H C}\left(T_{3}\right)$ such that $[A \mathbf{v}, \phi]$ is equal to the expression in (2.37) for every $\phi$ in $\mathfrak{F}\left(T_{3}\right)$.

Next, we observe that the following lemma holds:

Lemma 6. Let $A$ be the operator defined on $\mathfrak{H C}\left(T_{3}\right)$ by $(2.37)$, i.e., $[A \mathbf{v}, \boldsymbol{\phi}]$ equals the expression in (2.37) for every $\boldsymbol{\phi}$ in $\mathfrak{H C}\left(T_{3}\right)$. Then $A$ is a completely continuous operator.

The proof of Lemma 6 follows from Lemmas 1, 2, and 3 as in [2, p. 117]. We leave the details to the reader.

Next, we establish the following lemma:

Lemma 7. Let $A$ be the operator defined in Lemma 6. Then $[A \mathbf{v}, \mathbf{v}]=0$ for $\mathrm{v}$ in $\mathfrak{H}\left(T_{3}\right)$.

To establish Lemma 7, we first note the following Remark.

Remark 1. Let $g$ be in $L^{\gamma}\left(T_{3}\right), 1 \leqq \gamma<\infty$ and let $g(x, t)$ be defined as in (2.15). Then

$$
\int_{T_{3}}|g(x, t)-g(x)|^{\gamma} d x \rightarrow 0 \text { as } t \rightarrow 0 .
$$

The proof of Remark 1 follows easily from the proof of [3; Remark 2, p. 76].

Next, we say $g$ is in $W_{3 / 2}^{1}\left(T_{3}\right)$ if $g$ is in $L^{3 / 2}\left(T_{3}\right)$ and if there are functions $f_{k}, k=1,2,3$, in $L^{3 / 2}\left(T_{3}\right)$ such that

$$
\int_{T_{3}} g(x) \psi_{x_{k}}(x) d x=-\int_{T_{3}} f_{k}(x) \psi(x) d x \text { for all } \psi \text { in } \mathscr{D}\left(T_{3}\right) .
$$

We shall refer to $f_{k}(x)$ as $g_{x_{k}}(x)$. 
Proof of Lemma. Now let $\mathbf{v}$ be given in $\mathfrak{F}\left(T_{3}\right)$. Then we observe from Lemma 2 , Remark 1, and (2.38) that, for $j=1,2,3$,

$$
w_{j}=v_{j}^{2} \text { is in } L^{3}\left(T_{3}\right) \cap W_{3 / 2}^{1}\left(T_{3}\right)
$$

and

$$
w_{j x_{k}}=2 v_{j} v_{j x_{k}} .
$$

Next, we observe that for $t>0$,

$$
\int_{T_{3}} v_{k}(x, t) w_{j x_{k}}(x, t) d x=-\int_{T_{3}} v_{k x_{k}}(x, t) w_{i}(x, t) d x
$$

Since $v_{k}$ is in $L^{3}\left(T_{3}\right) \cap W_{3 / 2}^{1}\left(T_{3}\right)$, we have from (2.39), (2.41), and Remark 1 that

$$
\int_{T_{3}} v_{k}(x) w_{j x_{k}}(x) d x=-\int_{T_{3}} v_{k x_{k}}(x) w_{j}(x) d x .
$$

But then from this fact and (2.40), we conclude that

$$
2 \int_{T_{3}} v_{k}(x) v_{j}(x) v_{i x_{k}}(x) d x=-\int_{T_{3}} v_{k x_{k}}(x) v_{j}{ }^{2}(x) d x .
$$

Now from (2.37) and this last fact, we have that

$$
\begin{aligned}
(2 \pi)^{3}[A \mathbf{v}, \mathbf{v}] & =\sum_{j=1}^{3} \sum_{k=1}^{3} \int_{T_{3}} v_{k} v_{j} v_{j x_{k}} d x \\
& =-2^{-1} \sum_{i=1}^{3} \sum_{k=1}^{3} \int_{T_{3}} v_{j}^{2} v_{k x_{k}} d x=-2^{-1} \sum_{j=1}^{3} \int_{T_{3}} v_{i}{ }^{2}\left(\sum_{k=1}^{3} v_{k x_{k}}\right) d x=0
\end{aligned}
$$

and the proof of the lemma is complete.

Next, using (2.14), we set

$$
U_{i k i}(x)=\partial u_{k}^{i}(x) / \partial x_{i} \text { for } x \text { in } E_{3}-\bigcup_{m}\{2 \pi m\}
$$

and observe from (2.6) and (2.10) that

$$
U_{j k i}(x) \text { is in } C^{\infty}\left[E_{3}-\cup\{2 \pi m\}\right] \text {. }
$$

As a consequence of (2.11), (2.12), and (2.14), we conclude that there is a constant $\alpha^{\prime}$ such that for $i, j, k=1,2,3$,

$$
\left|U_{j k i}(x)\right| \leqq \alpha^{\prime}|x|^{-2} \text { for } x \text { in } B(0,2)-\{0\} .
$$

Observing that $U_{j k i}$ is periodic in $E_{3}-\bigcup_{m}\{2 \pi m\}$, we conclude from (2.44), (2.45), and [2; Lemma 5, p. 13] that the following holds:

Remark 2. Let $g(x)$ be in $L^{3 / 2}\left(T_{3}\right)$, and set

$$
f(x)=(2 \pi)^{-3} \int_{T_{3}} g(y) U_{i k i}(x-y) d y .
$$


Then $f$ is defined almost everywhere in $T_{3}$, and $f$ is in $L^{3}\left(T_{3}\right)$.

We leave the details of the proof of Remark 2 to the reader.

Next, we establish the following lemma:

Lemma 8. Let $\mathbf{f}=\left(f_{1}, f_{2}, f_{3}\right)$ where $f_{i}$ is in $L^{3 / 2}\left(T_{3}\right)$ and $\hat{f}_{i}{ }^{(}(0)=0$ for $j=1,2,3$. Set $\mathbf{v}=\left(v_{1}, v_{2}, v_{3}\right)$ where $v_{i}$ is defined almost everywhere in $T_{3}$ by (2.31). Then

$$
\mathbf{v} \text { is in } \mathfrak{H C}\left(T_{3}\right)
$$

and

$$
-\nu[\mathbf{v}, \boldsymbol{\phi}]=\sum_{j=1}^{3}(2 \pi)^{-3} \int_{T_{3}} f_{i} \phi_{j} d x \text { for } \boldsymbol{\phi} \text { in } \mathfrak{H C}\left(T_{3}\right) .
$$

Proof. From (2.31), we observe

$$
v_{j}^{\wedge}(m)=\sum_{k=1}^{3} u_{j}{ }^{k \wedge}(m) f_{k}^{\wedge}(m) .
$$

From the hypothesis of the lemma, (2.43), and Remark 2, and the fact that $\left|U_{j k i}{ }^{\wedge}(m)\right|=\left|m_{i} u_{k}{ }^{i \wedge}(m)\right|$, we have in particular that,

$$
\sum_{m \neq 0}\left|m_{i}\right|^{2}\left|u_{j}^{k \wedge}(m) f_{k}{ }^{\wedge}(m)\right|^{2}<\infty, \quad i, j, k=1,2,3 .
$$

We consequently conclude from (2.48) that

$$
v_{i} \text { is in }{ }_{0} W_{2}{ }^{1}\left(T_{3}\right), \quad j=1,2,3 .
$$

Next, for $t>0$, we set

$$
\begin{aligned}
& v_{j}(x, t)=\sum_{m}{v_{i}}^{\wedge}(m) e^{i(m, x)-|m| t}, \\
& f_{j}(x, t)=\sum_{m}{f_{i}}^{\wedge}(m) e^{i(m, s)-|m| t},
\end{aligned}
$$

and define $p(x, t)$ by $(2.32)$ with $f_{k}(y)$ replaced by $f_{k}(y, t)$. We also note from (2.48) that $v_{j}(x, t)$ is equal to the expression on the right hand side of the equality sign in (2.31) when $f_{k}(y)$ is replaced by $f_{k}(y, t)$. As a consequence we obtain from Lemma 5 that for $t>0$

$$
\sum_{k=1}^{3} \int_{T_{3}} v_{k}(x, t) \psi_{x_{k}}(x) d x=0, \text { for } \quad \psi \quad \text { in } \mathscr{D}\left(T_{3}\right) .
$$

Letting $t \rightarrow 0$ in (2.51) and using Remark 1, we conclude that

$$
\sum_{k=1}^{3} \int_{T_{3}} v_{k}(x) \psi_{x_{k}}(x) d x=0
$$

for $\psi$ in $D\left(T_{3}\right)$. But this implies that $\sum_{k=1}{ }^{3} v_{k x_{k}}=0$ and this fact in conjunction with (2.49) establishes (2.46). 
Next, we let $\phi$ be in $\mathfrak{H C}\left(T_{3}\right)$ and define $\phi_{i}(x, t)$ in a manner analogous to (2.50). Then $\phi_{i}(x, t)$ is in $\mathscr{D}\left(T_{3}\right) \cap \mathfrak{F}\left(T_{3}\right)$, and we conclude from (2.35) and (2.24) that

$$
\begin{aligned}
-\nu\left\langle v_{j}(x, t), \phi_{j}(x, t)\right\rangle & +(2 \pi)^{-3} \int_{T_{3}} p(x, t) \phi_{j x_{j}}(x, t) d x \\
& =(2 \pi)^{-3} \int_{T_{3}} f_{i}(x, t) \phi_{j}(x, t) d x .
\end{aligned}
$$

Summing over $j$ and using the fact that $\phi(x, t)$ is in $\mathcal{F C}\left(T_{3}\right)$, we conclude that

$$
-\nu \sum_{j=1}^{3}\left\langle v_{i}(x, t), \phi_{i}(x, t)\right\rangle=(2 \pi)^{-3} \sum_{j=1}^{3} \int_{T_{3}} f_{i}(x, t) \phi_{i}(x, t) d x .
$$

Observing from Lemma 2 that $\phi_{j}$ is in $L^{6}\left(T_{3}\right)$ and using Remark 1, we let $t \rightarrow 0$ in (2.52) and conclude that

$$
-\nu \sum_{j=1}^{3}\left\langle v_{i}(x), \phi_{j}(x)\right\rangle=(2 \pi)^{-3} \sum_{i=1}^{3} \int_{T_{3}} f_{j}(x) \phi_{i}(x) d x .
$$

This last fact in conjunction with (2.36) establishes (2.47), and the proof of the lemma is complete.

3. Proof of the necessary condition of the theorem. Let $Z \subset B(0,1)$ be a given closed set of positive Newtonian capacity. Also with no loss in generality, we can suppose that $Z$ is of 3-dimensional Lebesgue measure zero.

From [1, pp. 88-9] we obtain that there exists a non-negative Borel measure $\mu$ with the following properties:

$$
\begin{gathered}
\mu\left(T_{3}-Z\right)=0 ; \\
\mu(Z)=1 ; \\
f_{i}{ }^{\prime}(x)=\int_{Z}\left(x_{i}-y_{i}\right)|x-y|^{-3} d \mu(y), \text { for } x \text { in } E_{3}-Z,
\end{gathered}
$$

is in $L^{2}$ on compact subsets of $E_{3}$ for $j=1,2,3$.

We view $\mu$ as defined on $T_{3}$ and set

$$
\mu^{\wedge}(m)=(2 \pi)^{-3} \int_{T_{3}} e^{-i(m, x)} d \mu(x) .
$$

Next, with $q_{i}(x)$ defined by (2.3), we set

$$
f_{i}(x)=(2 \pi)^{-3} \int_{Z} q_{i}(x-y) d \mu(y) \text { for } x \text { in } T_{3}-Z
$$

and observe from (2.8) and (3.3) that

$$
f_{i} \text { is in } L^{2}\left(T_{3}\right) \text { for } j=1,2,3 .
$$

Also, from (3.5), we have that

$$
f_{i}^{\wedge}(m)=q_{i}^{\wedge}(m) \mu^{\wedge}(m) .
$$


Consequently from (2.3), (3.6), and (3.7), we have that

$$
\sum_{m \neq 0}\left|\mu^{\wedge}(m)\right|^{2}|m|^{-2}<+\infty \text {. }
$$

Next, with $D\left(T_{3}\right)$ defined by (2.30), we select a function $\lambda$ with the following properties:

(i) $\lambda$ is in $D\left(T_{3}\right)$;

(ii) $\lambda \geqq 0$;

(iii) $\int_{T_{3}} \lambda(x) d x=1$;

(iv) $\lambda(x)=0$ for $x$ in $B(0,3 / 2)$;

(v) $\lambda(x)=0$ for $x$ in $T_{3}-B(0,2)$.

Next, we define the Borel measure $\eta$ on $T_{3}$ by

$$
d \eta(x)=d \mu(x)-\lambda(x) d x .
$$

Consequently,

$$
\eta^{\wedge}(m)=\mu^{\wedge}(m)-\lambda^{\wedge}(m),
$$

and we have from (3.2), (3.8), (3.9), and (3.10) that

$$
\eta^{\wedge}(0)=0
$$

and

$$
K_{\eta}^{2}=\sum_{m \neq 0}\left|\eta^{\wedge}(m)\right|^{2}|m|^{-2}<+\infty .
$$

Next, we observe from $\left(2.14^{\prime}\right)$ that

$$
\left|u_{i}{ }^{\wedge}(m)\right| \leqq 2|m|^{-2} \text { for } m \neq 0 .
$$

Consequently, we have from (3.13) and (3.14) that

$$
\sum_{m \neq 0}\left|u_{i}{ }^{1 \wedge}(m) \eta^{\wedge}(m)\right|^{2}|m|^{2} \leqq 4 K_{\eta}{ }^{2}<+\infty .
$$

But then from (2.14') and (3.15), we obtain that

$$
\text { there exists } F_{j} \text { in }{ }_{0} W_{\mathbf{2}}{ }^{1}\left(T_{3}\right) \text { such that }{F_{i}}^{\wedge}(m)=u_{j}{ }^{1 \wedge}(m) \eta^{\wedge}(m) \text {. }
$$

Next, we note from $\left(2.14^{\prime}\right)$ and an easy calculation that

$$
\sum_{i=1}^{3} m_{j} u_{j}{ }^{\wedge}(m)=0 \text { for all } m \text {. }
$$

Consequently, we have from (3.16) and this last fact that

$$
\mathrm{F}=\left(F_{1}, F_{2}, F_{3}\right) \text { is in } \mathfrak{H C}\left(T_{3}\right) \text {. }
$$

Next, we let $A$ designate the completely continuous operator defined on $\mathfrak{F C}\left(T_{3}\right)$ in Lemma 6 , and we set

$$
A^{\prime} \mathbf{v}=\nu^{-1}[A \mathbf{v}+\mathbf{F}] \text { for } \quad \mathrm{v} \text { in } \oiiint\left(T_{3}\right) .
$$


Since $\mathbf{F}$ is in $\mathfrak{H C}\left(T_{3}\right)$, we conclude from (3.18) that

$A^{\prime}$ is a completely continuous operator on $\mathfrak{H C}\left(T_{3}\right)$.

Next, let $\xi$ be a real number with $0 \leqq \xi \leqq 1$ and suppose there is a $\mathbf{v}^{\xi}$ in $\mathfrak{H C}\left(T_{3}\right)$ such that

$$
\mathbf{v}^{\xi}=\xi A^{\prime} \mathbf{v}^{\xi}
$$

Then from (3.18), (3.20), and Lemma 7, we have

$$
\begin{aligned}
{\left[\mathbf{v}^{\xi}, \mathbf{v}^{\xi}\right] } & =\xi \nu^{-1}\left\{\left[A \mathbf{v}^{\xi}, \mathbf{v}^{\xi}\right]+\left[\mathbf{F}, \mathbf{v}^{\xi}\right]\right\} \\
& =\xi \nu^{-1}\left[\mathbf{F}, \mathbf{v}^{\xi}\right] .
\end{aligned}
$$

But then from (3.15) and (3.16), we have that $\left|\left[\mathbf{F}, \mathbf{v}^{\xi}\right]\right| \leqq 2(3)^{1 / 2} K_{\eta}\left[\mathbf{v}^{\xi}, \mathbf{v}^{\xi}\right]^{1 / 2}$. Since $0 \leqq \xi \leqq 1$, we conclude from (3.21) that

$$
\left[\mathbf{v}^{\xi}, \mathbf{v}^{\xi}\right]^{1 / 2} \leqq \nu^{-1} 2(3)^{1 / 2} K_{\eta} .
$$

From (3.19), (3.20), (3.22), and the Leray-Schauder principle [2, p. 32], we obtain that there exists a $\mathbf{v}$ in $\mathfrak{H C}\left(T_{3}\right)$ such that $A^{\prime} \mathbf{v}=\mathbf{v}$. From (3.18), we consequently conclude

$$
\text { there exists a } \mathbf{v} \text { in } \mathcal{H C}\left(T_{3}\right) \text { such that } \nu \mathbf{v}=A \mathbf{v}+\mathbf{F} .
$$

Next, we set

$$
g_{j}=\sum_{k=1}^{3} v_{k} v_{j x_{k}} \text { for } j=1,2,3
$$

and observe from (2.39) and (2.40) that

$$
g_{j} \text { is in } L^{3 / 2}\left(T_{3}\right) \text {, and } g_{i}{ }^{\wedge}(0)=0 \text {. }
$$

Next, we set

$$
V_{j}(x)=(2 \pi)^{-3} \sum_{k=1}^{3} \int_{T_{3}} u_{i}^{k}(x-y) g_{k}(y) d y,
$$

and observe from Lemma 8 that

$$
\mathbf{V}=\left(V_{1}, V_{2}, V_{3}\right) \text { is in } \mathfrak{H C}\left(T_{3}\right)
$$

and furthermore that

$$
\nu[\mathbf{V}, \boldsymbol{\phi}]=-\sum_{j=1}^{3}(2 \pi)^{-3} \int_{T_{3}} g_{j} \phi_{j} d x \text { for } \boldsymbol{\phi} \text { in } \operatorname{HC}\left(T_{3}\right) .
$$

In (2.41) and (2.42), we showed

(3.29) if $w$ and $h$ are in $L^{3}\left(T_{3}\right) \cap W_{3 / 2}{ }^{1}\left(T_{3}\right)$, then $\int_{T_{3}} w h_{x_{k}} d x=-\int_{T_{3}} w_{x_{k}} h d x$. 
Setting $h=v_{i}$ and $w=v_{k} \phi_{i}$, we conclude in particular from Lemma 2 and (3.29) that

$$
-\int_{T_{3}} v_{k} \phi_{j} v_{j x_{k}} d x=\int_{T_{3}} v_{j}\left[v_{k} \phi_{j x_{k}}+\phi_{i} v_{k x_{k}}\right] d x .
$$

But then we conclude from this last fact and (3.24) that

$$
-\int_{T_{3}} g_{i} \phi_{i} d x=\sum_{k=1}^{3} \int_{T_{3}} v_{j} v_{k} \phi_{j x_{k}} .
$$

Next, we obtain from (2.37), Lemma 6, (3.28) and (3.30) that

$$
\nu[\mathbf{V}, \boldsymbol{\phi}]=[A \mathbf{v}, \boldsymbol{\phi}] \text { for } \boldsymbol{\phi} \text { in } \mathfrak{H C}\left(T_{3}\right) .
$$

But then from (3.23) and (3.31), we have that

$$
\nu[\mathbf{V}, \boldsymbol{\phi}]=[\nu \mathbf{v}-\mathbf{F}, \boldsymbol{\phi}] \text { for } \boldsymbol{\phi} \text { in } \mathfrak{F}\left(T_{3}\right) .
$$

We conclude from (3.32) that

$$
\mathbf{v}=\mathbf{V}+\nu^{-1} \mathbf{F}
$$

Next, we define the functions $P(x)$ and $P^{\prime}(x)$ almost everywhere on $T_{3}$ as follows:

$$
\begin{aligned}
P(x) & =(2 \pi)^{-3} \sum_{k=1}^{3} \int_{T_{3}} q_{k}(x-y) g_{k}(y) d y, \\
P^{\prime}(x) & =(2 \pi)^{-3} \int_{T_{3}} q_{1}(x-y) d \eta(y) .
\end{aligned}
$$

From (2.8), we have that $q_{k}(x)=\mathbf{0}\left(|x|^{-2}\right)$ as $x \rightarrow 0$. We consequently conclude (as we did in Remark 2) from (3.25), (3.34), and [2; Lemma 5, p. 13] that

$$
P(x) \text { is in } L^{3}\left(T_{3}\right) \text {. }
$$

From (3.35), we have that $P^{\prime \wedge}(m)=q_{1}{ }^{\wedge}(m) \eta^{\wedge}(m)$. But then we obtain from (2.3) and (3.13) that $\sum_{m \neq 0}\left|P^{\prime \wedge}(m)\right|^{2}<+\infty$, and therefore that

$$
P^{\prime}(x) \text { is in } L^{2}\left(T_{3}\right) \text {. }
$$

Next, we set

$$
\eta(x, t)=\sum_{m \neq 0} \eta^{\wedge}(m) e^{i(m, x)-|m| t},
$$

and define $P(x, t)$ and $P^{\prime}(x, t)$ in a similar manner.

Then it follows from Lemma 4, (3.12), (3.16), and (3.26) that for $t>0$,

$$
\nu \triangle V_{j}(x, t)-P_{x_{i}}(x, t)=g_{i}(x, t), \quad j=1,2,3,
$$

and

$$
\begin{aligned}
& \nu \triangle F_{1}(x, t)-P_{x_{1}}{ }^{\prime}(x, t)=\eta(x, t) \\
& \nu \triangle F_{2}(x, t)-P_{x_{2}}{ }^{\prime}(x, t)=0 \\
& \nu \triangle F_{3}(x, t)-P_{x_{3}}{ }^{\prime}(x, t)=0 .
\end{aligned}
$$


Next, we set

$$
p(x)=P(x)+\nu^{-1} P^{\prime}(x) .
$$

Using the well-known fact that

$$
\lim _{t \rightarrow 0} \int_{T_{3}} \psi(x) \eta(x, t) d x=\int_{T_{3}} \psi(x) d \eta(x) \text { for } \psi \text { in } \mathbb{D}\left(T_{3}\right),
$$

we conclude from (3.33), (3.38), (3.39), and (3.40) that

$$
\begin{aligned}
& \int_{T_{3}}\left[\nu v_{1} \Delta \psi+p \psi_{x_{1}}\right] d x=\int_{T_{3}} \psi\left[\nu^{-1} d \eta(x)+g_{1} d x\right] \\
& \int_{T_{3}}\left[\nu v_{j} \Delta \psi+p \psi_{x_{j}}\right] d x=\int_{T_{3}} \psi g_{j} d x, \quad j=2,3, \text { for } \psi \text { in } \mathscr{D}\left(T_{3}\right) .
\end{aligned}
$$

Next, we set

$$
w_{i k}=v_{i} v_{k}
$$

Then it follows from (3.24) that $g_{j}=\sum_{k=1}{ }^{3} w_{j k x_{k}}$ and that $w_{j k}$ is in $L^{3}\left(T_{3}\right) \cap$ $W_{3 / 2}{ }^{1}\left(T_{3}\right)$. We consequently conclude from (3.29) that

$$
\int_{T_{3}} \psi g_{j} d x=-\sum_{k=1}^{3} \int_{T_{3}} w_{j_{k}} \psi_{x_{k}} d x \text { for } \psi \text { in } \mathscr{D}\left(T_{3}\right) \text {. }
$$

Now, let $B(y, r), r_{-}>0$, be an open 3-ball such that

$$
B(y, r) \subset B(0,3 / 2) \text {, }
$$

and let

$$
\zeta \text { be in } C_{0}^{\infty}[B(y, r)] \text {. }
$$

It is clear that there exists a $\psi$ in $D\left(T_{3}\right)$ such that

$$
\begin{aligned}
& \psi=\zeta \quad \text { in } B(y, r) \\
& \psi=0 \quad \text { in } \quad T_{3}-B(y, r) .
\end{aligned}
$$

We consequently conclude from (3.10), (3.11), and (3.41) through (3.46) that

$$
\begin{aligned}
& \int_{B(y, r)}\left[\nu v_{1} \Delta \zeta+p \zeta_{x_{1}}+\sum_{k=1}^{3} w_{1 k} \zeta_{x_{k}}\right] d x=\int_{B(y, r)} \nu^{-1} \zeta d \mu(x), \\
& \int_{B(y, r)}\left[\nu v_{j} \Delta \zeta+p \zeta_{x_{j}}+\sum_{k=1}^{3} w_{j k} \zeta_{x_{k}}\right] d x=0, \quad j=2,3, \\
& \text { for } \zeta \text { in } C_{0}^{\infty}[B(y, r)] .
\end{aligned}
$$

Recalling that $Z \subset B(0,1)$ and that $\mu$ has its support on $Z$, we conclude from (3.36), (3.37), (3.40), (3.42), (3.44), (3.47), and [6, Theorem 3] that

there exists $\mathbf{v}^{\prime}$ in $C^{2}[B(0,3 / 2)-Z]$ and $p^{\prime}$ in $C^{1}[B(0,3 / 2)-Z]$ such that the following holds: (i) $\left(\mathbf{v}^{\prime}, p^{\prime}\right)$ satisfies the equations in (1.3) in $B(0,3 / 2)-Z$; (ii) $\mathbf{v}^{\prime}=\mathbf{v}$ and $p^{\prime}=p$ almost everywhere in $B(0,3 / 2)-Z$. 
From (3.36). (3.37), and (3.40), we have that $p$ is in $L^{2}\left(T_{3}\right)$, and we know that $\mathbf{v}$ is in $\mathcal{H}\left(T_{3}\right)$. We consequently conclude from (3.48) that

$$
\left(\mathbf{v}^{\prime}, p^{\prime}\right) \text { meets conditions (1.1), (1.2), and }(1.3) \text { in } B(0,1)-Z \text {. }
$$

Suppose $Z$ were a removable set for stationary viscous incompressible flows. Then we would obtain from (3.49) that

$$
v^{\prime} \text { and } p^{\prime} \text { can be defined on } Z \text { so that }\left(\mathbf{v}^{\prime}, p^{\prime}\right) \text { meets conditions (1.1), }
$$
(1.2), and (1.3) in $B(0,1)$.

But then it is easy to see that (3.50) implies, in particular, the following fact:

$$
\begin{aligned}
\int_{B(0,1)}\left[\nu v_{1}{ }^{\prime} \Delta \zeta+p^{1} \zeta_{x_{1}}+\sum_{k=1}^{3} v_{1}{ }^{\prime} v_{k}{ }^{\prime} \zeta_{x_{k}}\right] d x & =0 \\
& \text { for } \zeta \text { in } C_{0}{ }^{\infty}[B(0,1)]
\end{aligned}
$$

Now recalling that by assumption, the 3-dimensional Lebesgue measure of $Z$ is zero, we see from (3.48) that $\mathbf{v}=\mathbf{v}^{\prime}$ and $p=p^{\prime}$ almost everywhere in $B(0,1)$. Consequently, using (3.42) and (3.44) and comparing (3.51) with the first equation in (3.47), we see that

$$
\int_{B(0,1)} \zeta d \mu(x)=0 \text { for } \zeta \quad \text { in } C_{0}^{\infty}[B(0,1)]
$$

But (3.52) contradicts (3.2). $Z$, therefore, is not a removable set for stationary viscous incompressible flows, and the necessary condition of the theorem is complete.

\section{REFERENCES}

1. L. Carleson, Selected problems on exceptional sets, Van Nostrand, Princeton, 1967.

2. O. A. Ladyzhenskaya, The mathematical theory of viscous incompressible flow, Revised 2nd ed., Gordon \& Breach, New York, 1969.

3. V. L. Shapino, Fourier series in several variables, Bull. Amer. Math. Soc. 70 (1964), 48-93.

4. - Characteristic planes and pointwise solutions of the heat equation, Indiana Univ. Math. J. 20 (1970), 115-133.

5. - Capacity and the nonlinear Navier-Stokes equations, SIAM J. Math. Anal. 4 (1973), 329-343.

6. - Isolated singularities for solutions of the nonlinear stationary Navier-Stokes equations, Trans. Amer. Math. Soc. 187 (1974), 335-363.

This research was sponsored by the Air Force Office of Scientific Research, Office of Aerospace Research, USAF, under Grant No. AFOSR 73-2456. The United States Government is authorized to reproduce and distribute reprints for Governmental purposes notwithstanding any copyright notation hereon. 